\title{
On some Spectral Properties of TanDEM-X Interferograms over Forested Areas
}

\author{
Francesco De Zan, Gerhard Krieger and Paco López-Dekker
}

\begin{abstract}
This letter reports about some obervations over rainforest (in Brazil and Indonesia), where the spectra of TanDEM-X interferograms show distinct features, almost a signature, which is explained and modelled in terms of the scattering properties. Supported by comparisons with simulations, the observations exclude any homogeneous, horizontally-layered forest; instead, they are compatible with a model with point scatterers clustered in clouds. Such a model, with high extinction and large gaps that allow significant penetration, is able to explain to a good degree the observations.
\end{abstract}

Index Terms-SAR interferometry

\section{INTRODUCTION}

Since the TanDEM-X satellite has been positioned in a close formation with its twin TerraSAR-X, the two have acquired thousands of SAR scenes in a bistatic configuration. The main goal of the mission is to produce a global digital elevation model (DEM) with unprecedented accuracy. Moreover the collected data constitute a unique dataset with a lot of additional potentialities.

The single-pass X-band interferometric pairs have a high resolution, being typically acquired with a $100-\mathrm{MHz}$ bandwidth. Thanks to a maximum along-track baseline in the order of 1 kilometer or less, the resulting interferograms are typically almost immune to temporal decorrelation[1].

The formation is tighly controlled and the baselines result in heights of ambiguity of a few dozen meters. For monitoring and calibration purposes some areas are acquired repeatedly with different configurations, opening the possibility to interesting studies.

Tropical rainforests are one exception to the generally high coherence found in TanDEM-X data. For instance Fig. 1 displays the coherence of acquisition \#1001508, which was taken over Brazil on October 23rd, 2010. The height of ambiguity is $25 \mathrm{~m}$ and the coherence allows to clearly distinguish forested areas and clearcuts. The coherence over the forest is just 0.25 0.30 and the corresponding phase can be difficult to unwrap.

\section{SPECTRA AND INTERPRETATION}

The interferometric coherence is the most common measure of the interferometric properties of two SAR images. It is well known to be linked to the target structure in the elevation direction. Additional information on the second-order statistics

The authors are with the Microwaves and Radar Institute of DLR (German Aerospace Center), Oberpfaffenhofen, D-82230 Wessling, Germany. email:francesco.dezan@dlr.de

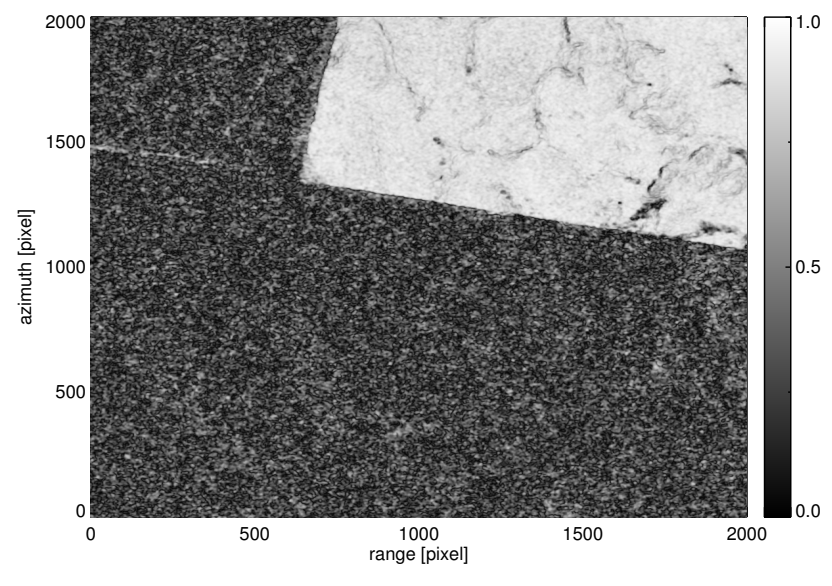

Fig. 1. A detail (about $4 \mathrm{~km} \times 4 \mathrm{~km}$ ) of the coherence map of TanDEM-X acquisition \#1001508. The height of ambiguity is $25 \mathrm{~m}$. The clearcuts have a very high coherence, whereas the forested areas have a low coherence, around $0.25-0.30$ (window size of 120 samples).

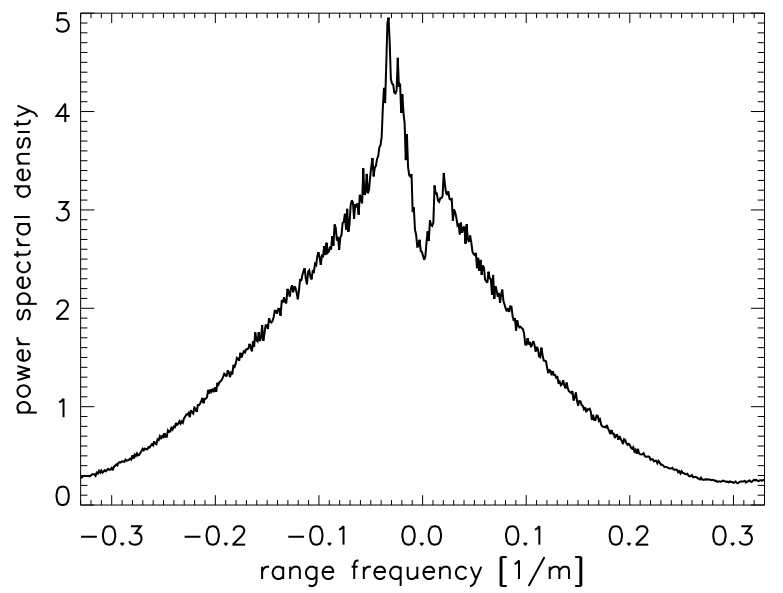

Fig. 2. The range spectrum of an interferogram (acquisition \#1001508) over Brazilian rainforest. The spectrum is averaged over 2000 azimuth lines. The height of ambiguity is $25 \mathrm{~m}$.

of the interferogram is given by its range spectrum:

$$
S\left(f_{r}, a\right)=\mathrm{E}\left[\left|\int y_{m}(r, a) y_{s}^{*}(r, a) e^{-j 2 \pi f_{r} r} d r\right|^{2}\right] .
$$

Here $y_{m}$ and $y_{s}$ are the master and the slave images, $r$ and $a$ the range and azimuth coordinates, $f_{r}$ the range frequency. The spectral analysis can be performed also in the azimuth 
direction, but this is not done in this paper. An estimate of the range spectrum can be obtained by averaging across azimuth many range periodograms.

The analysis of the periodograms of TanDEM-X bistatic interferograms over Brazilian and Indonesian rainforests shows some particular features, which were entirely unexpected and proved remarkably stable under a variety of conditions (different sites, incidence angles, etc.). Two distinct peaks are recognizable (Fig. 2), while around the zero frequency there is a power dip. The higher peak corresponds to the flatEarth and, if we had flattened the interferogram, it would be translated to zero. For this analysis we prefer not to flatten the interferograms, for flattening makes the physical interpretation slightly more complex.

To explain the range spectrum of the interferogram we note first that each frequency corresponds to a slope in the scene [2], and thus the spectrum is closely related to the slope distribution in the scene (Woodward's theorem for frequencymodulated signals). This interpretation is compatible with the high extinction rate expected at $\mathrm{X}$ band: it seems reasonable for the scatterers to be organized along surfaces with different slopes. For longer wavelengths, a model based on volumetric targets could be more appropriate. The relation between slopes and frequencies is the following (bistatic case, see also [3]):

$$
f=-\frac{f_{0} B_{n}}{2 R_{0} \tan \left(\vartheta_{i n c}\right)} .
$$

Here $f_{0}$ is the central frequency, the normal baseline is $B_{n}, R_{0}$ is the range distance and $\vartheta_{i n c}$ is the (local) incident angle, that is the local slope referred to the line of sight. Figure 3 shows this relation for some typical TanDEM-X system parameters.

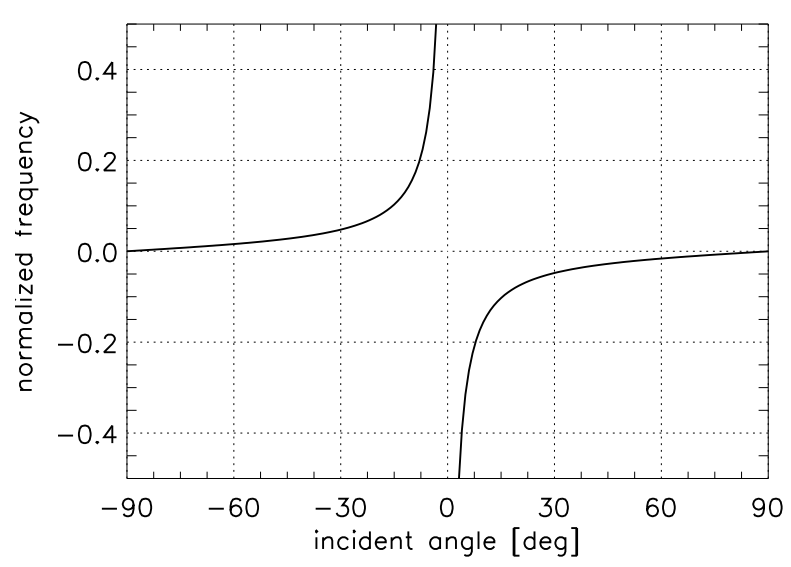

Fig. 3. The relation between incident angles and frequency for a realistic TanDEM-X example (400m normal baseline).

According to this interpretation, the two peaks in Fig. 2 correspond to physical slopes in the scene which are either regularly imaged or in lay-over conditions. With our baseline conventions the positive frequencies map to slopes in layover. The zero-frequency components map to slopes aligned with the line of sight. At the edges of the spectrum we find foreshortened slopes (around normal incidence). One could imagine that trunks and the vertical parts of the canopies are imaged upside down, whereas the horizontal parts of the canopies and the ground are imaged normally.

However, the correspondence between slopes and frequencies is not simple and several aspects have to be taken into account to explain the observed spectra:

1) The relation between slopes and frequencies is nonlinear, so that slopes at $90 \mathrm{deg}$ incidence are packed closer together in the spectrum (in relative terms, compared to slopes facing the radar). The Jacobian of the transformation is $\sin ^{2}\left(\vartheta_{\text {inc }}\right)$. This term has the effect of concentrating the spectrum around low frequencies.

2) The slopes facing the radar are better illuminated than the slopes oriented along the line of sight. Indeed at grazing incidence the surface will end up being in the shadow of itself, and almost no energy will be backscattered. The result on the spectrum is the presence of a hole around frequency zero and the appearance of two distinct peaks.

3) The sloped surfaces have not infinite length: discontinuities will broaden the spectral peaks.

4) The presence of topography will have an impact on the shape of the spectrum: it is expected that the peak corresponding to the flat-Earth frequency will also be broadened.

5) Variations of the geometry across the swath will also change the relation between slopes and frequencies: for instance they would broaden the flat-Earth peak, depending on the range size of the estimation window.

6) The spectral weighting of the original images affects the shape of the flanks of interferogram spectrum. The latter is rougly a convolution of the former, for the decorrelated components. This generates a noise pedestal which masks information about foreshortened slopes.

\section{Simulations}

Since it is difficult to describe analytically all the mentioned aspects, and in order to support the explanation of the observed spectral signature, we conducted some 2-D simulations (the azimuth dimension is ignored) based on clouds of point targets. In analyzing these models, we are more interested in the general behaviors of the predicted spectra than in fine-tuning the model parameters to perfectly match the observations.

The first model we present (Fig. 4) is a random layered model, extending up to 45 meters. The model predicts correctly the position of the flat-Earth peak, but cannot explain the secondary peak in the spectrum, since it does not include scatterers extended in the vertical direction.

The second model (Fig. 5) has ground scatterers and vertical scatterers which could represent the trunks. The trunks are 15 to 30 meter high. As it was suggested by one anonymous reviewer, such a model would explain the main features of our spectra, namely the two peaks, their positions, and the dip between them.

The third model consists of a random distribution of targets clustered in areas delimited by ellipses (Fig. 6, with major axes ranging from 10 to $15 \mathrm{~m}$, minor axes being half of the major axes). The ellipses are intended to correspond to the tree 

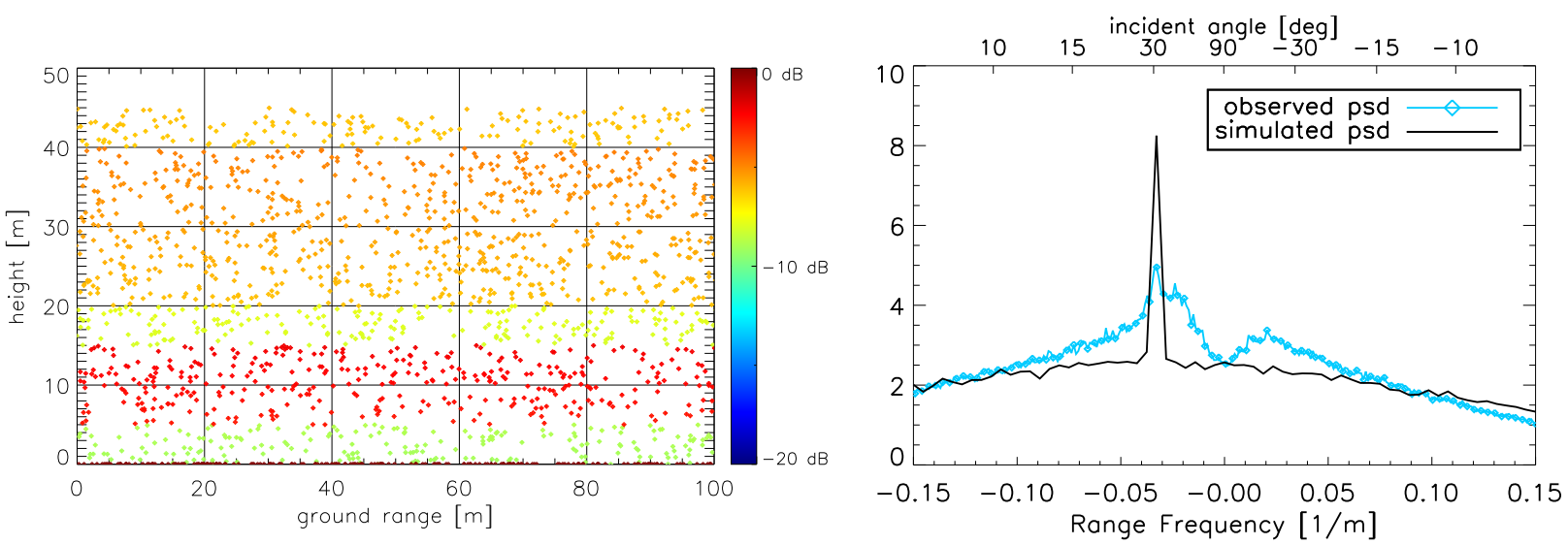

Fig. 4. Left: A realization of a layered forest model. Right: The observed range spectrum (blue) of an interferogram (acquisition \#1001508) over Brazilian rainforest and a simulated spectrum (black).
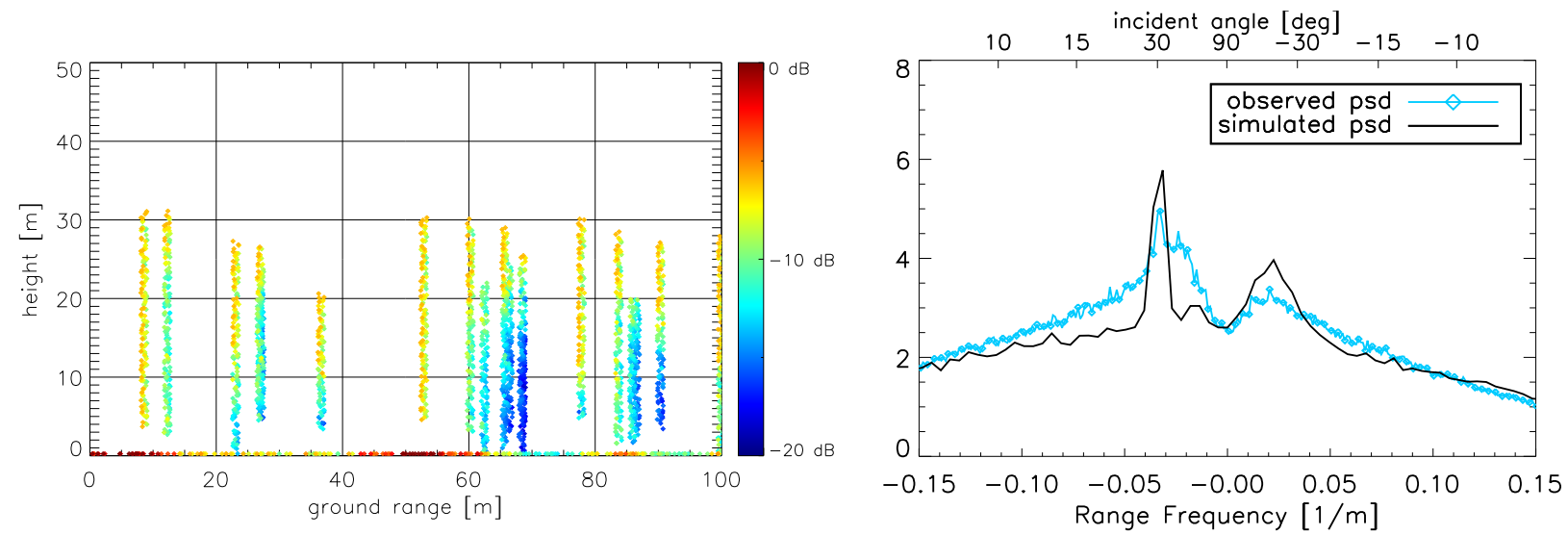

Fig. 5. Left: A realization of a 'matchstick' forest model. Right: The observed range spectrum (blue) of an interferogram (acquisition \#1001508) over Brazilian rainforest and a simulated spectrum (black).
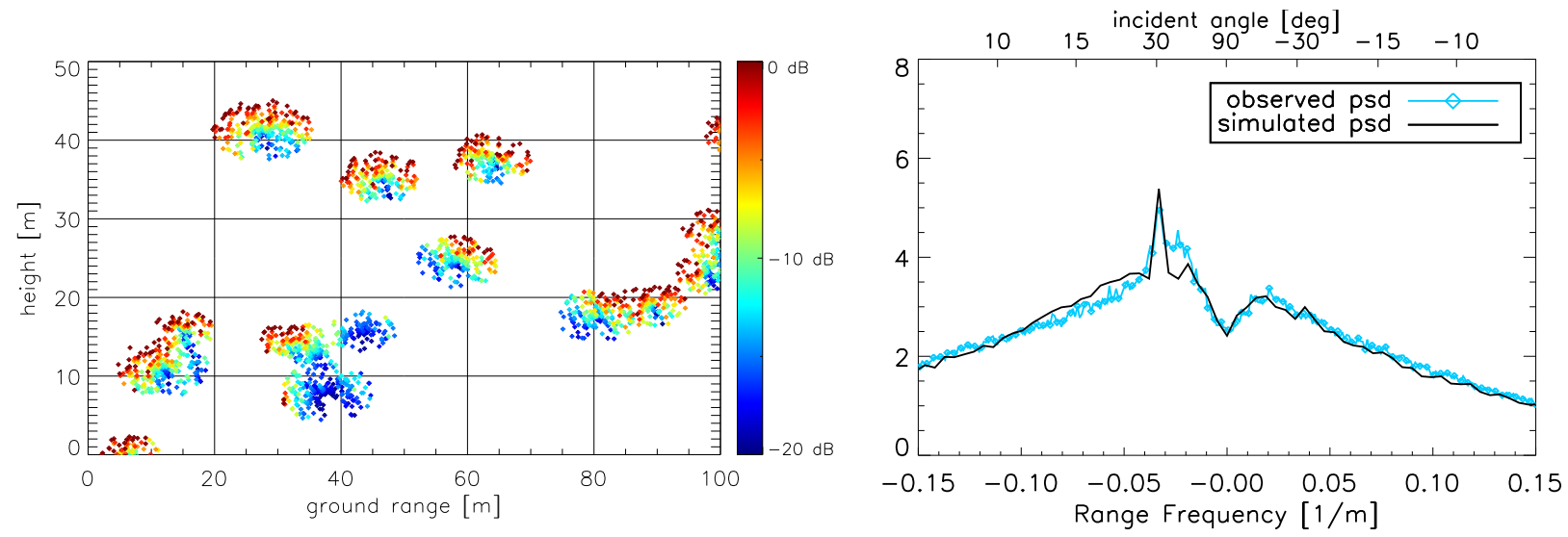

Fig. 6. Left: A realization of the forest model with scatterers within ellipses. Note the shadowing effect between scatterers. Right: The observed range spectrum (blue) of an interferogram (acquisition \#1001508) over Brazilian rainforest and a simulated spectrum (black).

crowns or large branches. They are randomly placed from the ground surface up to the maximum forest height $(45 \mathrm{~m}$ in the example).

The crucial part of the model is the presence of an extinction mechanism. Scatterers which are not in full visibility are attenuated according to the number of scatterers which obscure them in the line of sight. Depending on the extinction coefficient, the scattering character goes from volume-like (low extinction) to surface-like (high extinction). A similar model, with parameters partially derived from field observations, was used for example in [4].

Although the match between the spectra in Fig. 6 is not 
perfect, it is enough to conclude that no further cause needs to be invoked: the main interferogram spectral properties can be explained on the basis of a complex but realistic forest structure. The change in the spectral shapes is well-behaved w.r.t. changes in the parameters, and it is possible to get similar shapes with different forest heights, by changing the extinction, the density of the ellipses etc.

\section{DISCUSSION}

From the simulations it is clear that the spectral properties of the interferograms are related to the forest structure. In order to explain the second-order statistics of the interferograms it is necessary to include some lateral variations in the model: any layered model, homogenous in the horizontal dimension, will yield only one peak in the inteferogram spectrum. This can be justified noting that a layered model is a superposition of many horizontal surfaces. Since the scatterers are independent, the resulting expected interferogram is the sum of the expected interferograms corresponding to each individual surface, each one with a different intensity and phase, due to the original brightness of the surface and its height. Finally, the resulting range spectrum will have the same monomodal shape of the spectrum of a flat surface.

The 'matchstick' model (Fig. 5), with ground scatterers and trunks, is already able to account for the two peaks, one peak for the ground and one peak for the trunks. The best fit so far was obtained with a model comprising elliptical clouds of scatterers and strong extinction (Fig. 6), so that almost only the targets illuminated on the surface of the ellipses effectively contribute to the signal.

It is necessary to point out that the simulations presented in Figures 4-6 do not constitute an attempt to a full inversion of forest parameters, but a contribution to the discussion on forest models and a first-order explanation of our observations. Whether or not we can really extract useful information from those spectra, is still to be proven. For sure several models would give the same power spectra (the phases carry additional information that is lost in the moduli).

The problem of fitting the spectral shapes can be considered a distinct problem from the one of fitting the coherences, the latter concerned with vertical structure and the former with horizontal structure. Of course it will be desirable to have a single 2-D scattering model able to simultaneously predict the spectra and the coherences.

Discussions about the appropriate forest model for SAR interferometry are not new. A homogenous water-cloud model has been often adopted for the canopies; it has been observed that such a model requires very low extinctions to predict the low interferometric coherences found in real data [5]. The works of Treuhaft and Rodriguez on coherence statistics and multi-frequency analyses already dealt with some shortcomings of such a simple homogeneous model. Significant penetration due to large gaps in the canopy has been often suggested in the past [6], [7], [8] to explain the apparent low extinction, and it is also useful today to account for TanDEM$\mathrm{X}$ interferogram spectra. Direct observation of the ground through gaps in the canopy could also be inferred from the examination of scatterometer data, for instance in [9].

\section{Spatial AVERAging AND MUlti-BASEline}

One way to improve the unwrapping quality over rainforest is to ensure that the acquisitions satisfy a minimum height of ambiguity such that unwrapping difficulties are avoided. This solution was the one adopted for the TanDEM-X mission. Another solution, perhaps the easiest, is to enlarge the averaging window to recover a coarse but reliable phase.

However, one has to be aware that spatial averaging (multilooking) in the rainforest case has different implications compared to, e.g., filtering a decorrelated interferogram that images a smooth surface. In the latter example the spectral components outside the main peak have no physical meaning, i.e. they do not correspond to physical slopes, and it is meaningful to classify them as noise and safe to suppress them. In the forest case, instead, some of the energy in the secondary peak does indeed correspond to physical slopes (e.g. vertically aligned scatterers). Spatial averaging will implicitely reject signal components which surely carry some information.

Since the results point to the presence of surface scatterers, it might be true that the problem is closer to being a surface unwrapping problem than a pure tomographic problem, at least for the high frequency and large bandwidth used. In this case, as for DEM retrievals, one could try using a combination of a large baseline (for accuracy) and a small one (for unwrapping). Using two baselines carries the risk that the target undergoes changes in the meantime: this can be avoided with TanDEM$\mathrm{X}$ by making alternating bistatic acquisitions which give simultaneously three images - two monostatic and one bistatic - from which two different baselines can be obtained.

In case that some layover is anyway present, and more than two images are available, one should consider tools like compressive sensing or model-based layover solutions, which can take advantage of the sparsity of the scatterers in the tomographic dimension [10]. These tools are already used for urban landscapes, which, as a reviewer has noted, could match closely forest scenarios from the radar point of view.

\section{CONCLUSIONS AND FUTURE INVESTIGATIONS}

The spectral analysis of TanDEM-X interferograms gives some new insight into the forest structure, as seen at $\mathrm{X}$ band. Simulations show the inadequacy at the sensor's high resolution of a simple model consisting of horizontal layers to explain radar observables: the system is sensitive to horizontal inhomogeneities. A structure consisting of clouds of scatterers, with gaps and extinction, is able to account for the main interferogram spectral properties.

This is an indication that at $100-\mathrm{MHz}$ bandwidth the (second-order) coherence is not a complete statistical description of the interferogram and therefore one should be aware that spatial averaging reduces the information contained in the interferogram.

Due to the surface-like nature of the target revealed by spectral analysis, multi-baseline approaches could be tried to reconstruct the surface at high resolution, by solving a multibaseline unwrapping problem or low-order layover.

Further investigations will clarify whether the found correlation properties are consistent across polarizations and whether 
they are even more pronounced for higher resolution sensors. Future studies are needed to attempt to extract information about the forest structure from the interferogram spectra, for example the level of sparsity.

\section{ACKNOWLEDGEMENT}

The TanDEM-X project is partly funded by the German Federal Ministry for Economics and Technology (Förderkennzeichen 50 EE 1035). The authors would also like to thank the anonymous reviewers for their valuable work.

\section{REFERENCES}

[1] G. Krieger, A. Moreira, H. Fiedler, I. Hajnsek, M. Werner, M. Younis, and M. Zink, "TanDEM-X: a satellite formation for high-resolution SAR interferometry," Geoscience and Remote Sensing, IEEE Transactions on, vol. 45, no. 11, pp. 3317-3341, Nov 2007.

[2] A. Monti Guarnieri, "SAR interferometry and statistical topography," Geoscience and Remote Sensing, IEEE Transactions on, vol. 40, no. 12, pp. 2567-2581, Dec 2002.

[3] F. Gatelli, A. Monti Guarnieri, F. Parizzi, P. Pasquali, C. Prati, and F. Rocca, "The wavenumber shift in SAR interferometry," Geoscience and Remote Sensing, IEEE Transactions on, vol. 32, no. 4, pp. 855-865, July 1994.

[4] C. Varekamp and D. H. Hoekman, "High-resolution InSAR image simulation for forest canopies," Geoscience and Remote Sensing, IEEE Transactions on, vol. 40, no. 7, pp. 1648-1655, Jul. 2002.

[5] P.A. Rosen, S. Hensley, I.R. Joughin, F.K. Li, S.N. Madsen, E. Rodriguez, and R.M. Goldstein, "Synthetic aperture radar interferometry," Proceedings of the IEEE, vol. 88, no. 3, pp. 333 -382, Mar 2000.

[6] J. Askne et al., "C-band repeat-pass interferometric SAR observations of the forest," Geoscience and Remote Sensing, IEEE Transactions on, vol. 35, no. 1, pp. 25-35, Jan. 1997.

[7] J. Askne at al., "Multitemporal repeat-pass SAR interferometry of boreal forests," Geoscience and Remote Sensing, IEEE Transactions on, vol. 41, no. 7, pp. 1540 - 1550, Jul. 2003.

[8] R. Treuhaft et al., "Vegetation profiles in tropical forests from multibaseline interferometric synthetic aperture radar, field, and lidar measurements," J. Geophys. Res., vol. 114, Dec 2009.

[9] J. Hyyppa and M. Hallikainen, "A helicopter-borne eight-channel ranging scatterometer for remote sensing. II. Forest inventory," Geoscience and Remote Sensing, IEEE Transactions on, vol. 31, no. 1, pp. 170 -179, Jan 1993.

[10] X. Zhu and R. Bamler, "Tomographic SAR inversion by $L_{1}$-norm regularization - the compressive sensing approach," Geoscience and Remote Sensing, IEEE Transactions on, vol. 48, no. 10, pp. 3839 -3846, Oct 2010. 